\title{
Amodal completion and visual holes (static and moving)
}

\author{
Marco Bertamini ${ }^{\mathrm{a}, *}$, Johan Hulleman ${ }^{\mathrm{b}}$ \\ ${ }^{a}$ School of Psychology, Eleanor Rathbone Building, University of Liverpool, Liverpool L69 7ZA, United Kingdom \\ ${ }^{\mathrm{b}}$ Department of Psychology, University of Hull, Cottingham Road, Hull HU67RX, United Kingdom
}

Received 21 October 2005; received in revised form 27 April 2006; accepted 28 April 2006

Available online 14 August 2006

\begin{abstract}
Occlusion is a frequent occurrence in a cluttered world of opaque objects. Often information about the shape of partly occluded objects can be gathered from the visible portion of the object and in particular its contours. Here we address the case where a region of a surface is visible exclusively through an aperture (visual hole). We make several observations about the grouping of surface regions visible through holes, and the appearance of moving objects and holes. These observations support the view that holes are shape properties of the object-with-hole.
\end{abstract}

(c) 2006 Elsevier B.V. All rights reserved.

PsycInfo: 2323

Keywords: Visual holes; Amodal completion; Figure-ground organisation; Shape

\section{Introduction}

If one accepts that the fundamental problem of vision is the fact that visual images have many possible interpretations, then it is clear that the perception of partly occluded objects is not a special case. Visual scenes in which occlusion is present are the norm rather than

\footnotetext{
* Corresponding author. Tel.: +44 151794 2954; fax: +44 1517942945.

E-mail addresses: M.Bertamini@liverpool.ac.uk (M. Bertamini), J.Hulleman@hull.ac.uk (J. Hulleman).

URL: http://www.liv.ac.uk/VP/ (M. Bertamini).
} 
the exception and because of occlusion the visual system is dealing with incomplete shape information. In turn, this way of setting the problem explains why interest in how visual objects are perceived behind occluders has remained strong for several decades.

A necessary start for the study of occlusion is phenomenology. In their essay, Michotte, Thinès, and Crabbé (1967) discuss several phenomena and provide a useful terminology. The term screen ("écran") for the occluder has not been widely adopted, but the term amodal ("donné amodal" and "complément amodal"), for the completion of visual structure without the sensation of contour or surface, has entered the vocabulary of vision scientists around the world.

The amodal completion literature has focussed on processes of extrapolation or interpolation. Much work has tested how the amodally perceived portion relates to the visible portion of an object. Contours clearly play a central role here, both locally and at a global level (Fantoni \& Gerbino, 2003; Kellman, Guttman, \& Wickens, 2001; Shipley \& Kellman, 1994; van Lier \& Wagemans, 1999). Recently, Fantoni, Bertamini, and Gerbino (2005) and Fulvio and Singh (2006) have studied the influence of some neglected factors, i.e., convexity and medial axis. Even in such cases, the information comes from the contours of the occluded object.

In this paper, we focus on a special case of completion in which there is no information about the contours of the occluded object. That is the case when the occluder and the occluded outline do not intersect, and this happens in two situations (Fig. 1). The first situation is when the occluder (figure) is simply covering a portion of uniformly textured surface (ground). Imagine a coin on a table with the table continuing its existence underneath the coin ("permanence de continuité", Michotte, 1950). The second situation is the complement

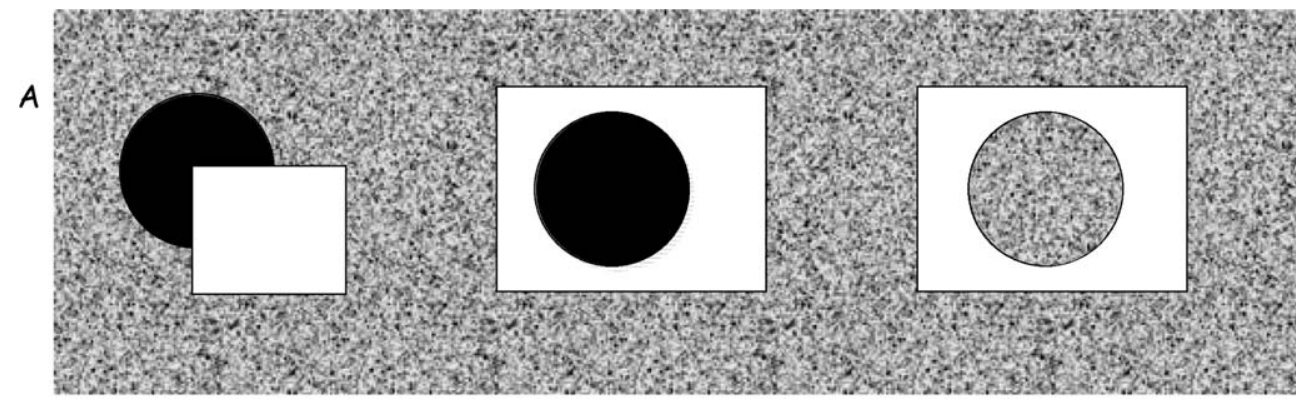

B

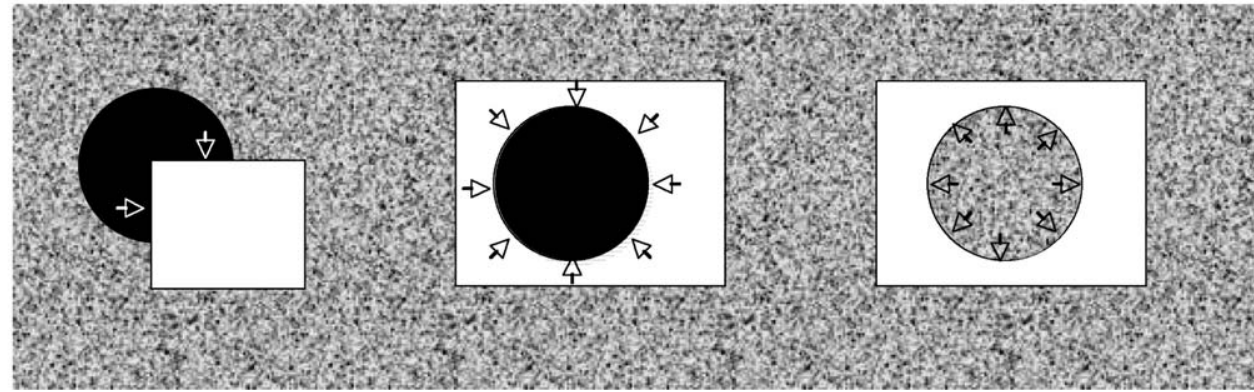

Fig. 1. (A) Three types of amodal completion. From left to right: information about the contour available; no information about the contour available in the presence of an object; no information about the contour available in the presence of the hole. (B) Direction of amodal completion is indicated by the arrows. 
of the first: a region of background seen through a hole in the foreground. The closed contour can be identical in both situations, for example a circle. The difference between a figure and a hole is in the figure/ground organisation, and this difference has fundamental implications for amodal completion. In the case of the circular figure, the surface extends inwards; in the case of the circular hole, the surface extends outwards. This type of amodal perception is, therefore, completely distinct from issues of contour interpolation. For the figure no new contours need to be involved, since both the outside contours of the figure (coin) and the outside contour of the background (table) are given. For the hole though, because the background surface extends beyond the visible region, this surface needs to stop at an invisible boundary. However, there is no logical reason to expect the invisible boundary to be related to the inside or outside contours of the occluder (the object-with-hole).

What happens if relative motion is introduced? When an object moves there is deletion of background information at the leading edge of the object, and accretion of background information at the trailing edge of the object (Gibson, Kaplan, Reynolds, \& Wheeler, 1969). When the moving occluder is an object-with-hole, the roles of leading and trailing edge are reversed: accretion at the leading edge of the hole and deletion at the trailing edge of the hole (Fig. 2). Although this would seem a difference between a moving object and a moving object-with-hole, they are similar with respect to the relation between accretion/ deletion and which side of the contour is in the foreground: when the side in the foreground is in front in terms of motion (i.e., trailing edge of the object, leading edge of the hole) there is accretion, when it is back in terms of motion (leading edge of the object, trailing edge of the hole) there is deletion. So, if the figural side owns the contour, accretion and deletion are similar for moving objects and moving objects-with-hole.

The claim that a figure owns the contour is a standard tenet in the figure-ground literature (e.g., Kanizsa, 1979; Nakayama, Shimojo, \& Silverman, 1989) and contour ownership affects many percepts, for instance, apparent motion (Shimojo \& Nakayama, 1990). However, there have been suggestions that a hole might share the ownership of the border

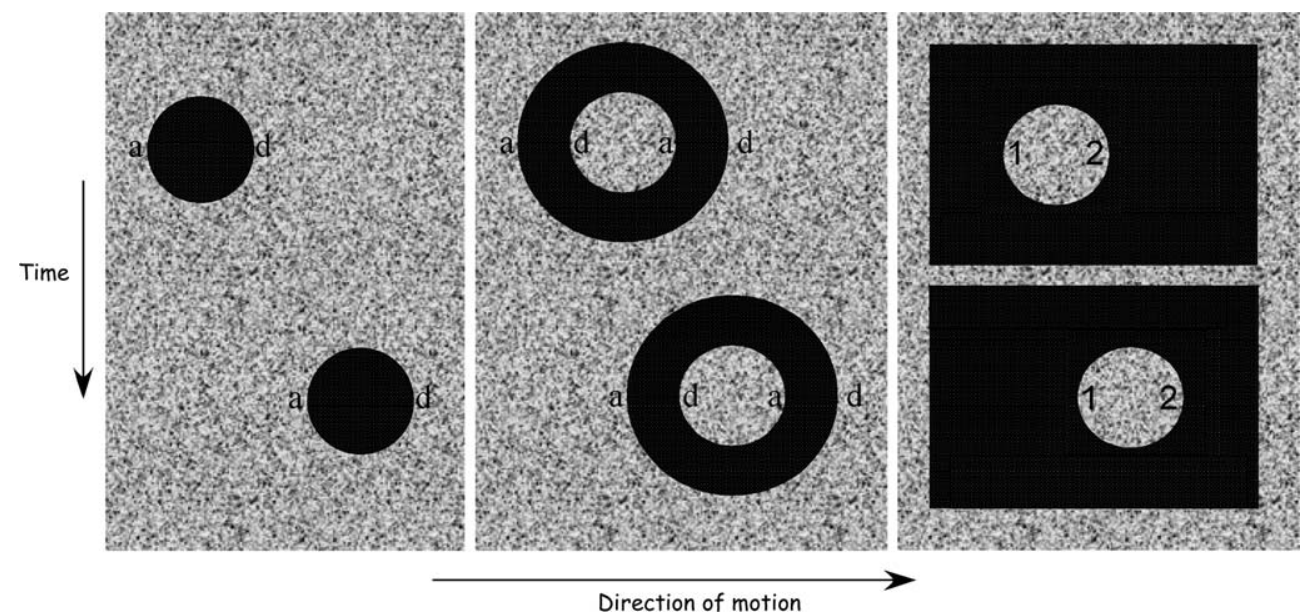

Fig. 2. Accretion and deletion of texture for a moving object, a moving object with a hole and a moving hole, respectively. Explanation of codes: (a) accretion of background texture; (d) deletion of background texture; (1) deletion of background texture and accretion of foreground texture; (2) accretion of background texture and deletion of foreground texture. 
between hole and object-with-hole (e.g., Palmer, 1999; Peterson, 2003), imparting objectlike properties like shape to it. One way to test contour ownership is to make the motion of the hole independent of the motion of the object-with-hole (Fig. 2). In this case, the hole changes the position on the object-with-hole. In addition to the accretion of background, there is also deletion of foreground at its leading edge (as the hole occludes parts of the object-with-hole that were previously unoccluded, while at the same time unoccluding parts of the background that were previously occluded, or, put differently, as the hole moves to a new position on the object-with-hole, the foreground texture that was visible before the hole arrived is replaced by the background texture that is visible through the hole) and there will be accretion as well as deletion at its trailing edge (parts of the objectwith-hole become unoccluded again if the hole has passed, while at the same time the object-with-hole occludes parts of the background previously visible through the hole). This gives the leading and trailing edges of the moving hole accretion/deletion characteristics similar to that of a moving object. We reasoned that if the contour belongs to the hole, participants should still be able to see the moving hole as a hole because the relation between the contour and accretion/deletion has not changed on the inside of the hole. The only change is that the hole is moving independently now, rather than together with the object-with-hole. If, however, the contour belongs to the object-with-hole, there should be a change in the perception of the moving hole, because accretion and deletion have been introduced on the side of the contour where there previously were none.

We discuss perception of visual holes more generally in Section 2. In Section 3, we look in more detail at what happens when holes move. In the final section, we discuss the implications of our findings for what would happen when holes take part in dynamic events like the tunnel effect (Burke, 1952) and a causality event (Michotte, 1946).

Since Michotte's time new methodologies have been developed, to the point that phenomenological reports are now rarely seen in research articles. However, some authors have recently shown renewed interest in some versions of the phenomenological approach (Kubovy \& Gepshtein, 2003). The approach in this paper is to analyse some critical stimuli, in the sense that observers are faced with interesting alternatives when viewing such stimuli. We have also presented these stimuli to naive observers and we analyse both their free descriptions and their forced-choice descriptions. This method has limitations and needs complementing, but important insights emerge from these data.

\section{Visual holes and amodal completion}

Holes have fascinated people for many reasons. Some authors have focused on the conditions under which we perceive holes as such (e.g., Bozzi, 1975; Nelson \& Palmer, 2001), some authors have looked at holes from a philosophical standpoint (Casati \& Varzi, 1994), some authors have used them to study structural shape (e.g., Bertamini \& Croucher, 2003), border ownership (e.g., Qiu \& von der Heydt, 2005), perception of depth and slant (e.g., Gillam \& Cook, 2001) and surface integration (Yin, Kellman, \& Shipley, 2000). ${ }^{1}$

\footnotetext{
${ }^{1}$ Not all authors refer to visual holes as such, other terms include "window" and "aperture". Window is not an ideal term, because it also refers to an everyday object. Aperture on the other hand is a precise term that is widely used in the study of perception, as in the case of the aperture problem (Wuerger, Shapley, \& Rubin, 1996). Visual hole, however, is the term that more clearly implies closure of the contour. For more on terminology, see Bertamini (in press).
} 


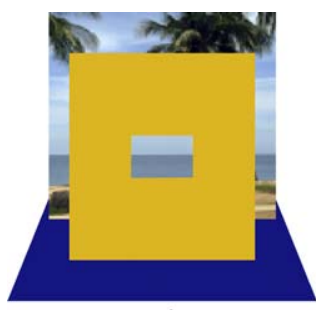

A

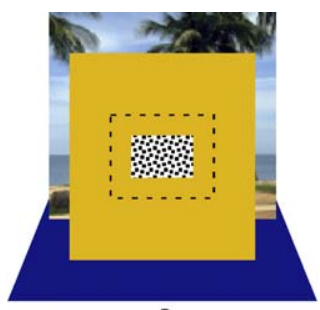

B

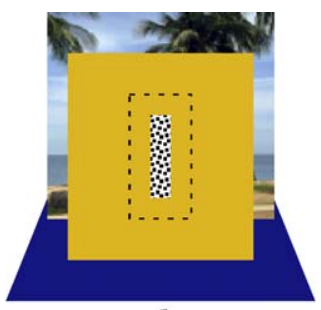

C

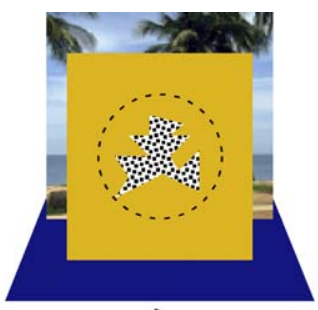

D

Fig. 3. A classification of different types of occlusions in the presence of a visual hole. (A) A region of background visible through a hole is closely related (grouped) with the background visible beyond the orange occluder. In (B) and (C), the dotted surface is only visible through the hole. Under pictorial conditions, this region would be perceived as a figure. The only difference between $\mathrm{B}$ and $\mathrm{C}$ is the orientation of the hole, but this may in turn affect what is amodally perceived, as illustrated by the dashed lines. A hole region high in complexity may lead to the perception of a less complex surface beyond the hole (D). (For interpretation of the references to colour in this figure legend, the reader is referred to the Web version of this article.)

Bertamini (in press) has argued that holes are ideal stimuli for the study of figureground organisation. The reason is that a pure figure-ground reversal turns a closed contour from a visual object into a visual hole and vice-versa (holes have been used for such purpose in Bertamini \& Croucher, 2003; Bertamini \& Mosca, 2004; Bertamini \& Lawson, 2006). With respect to the conditions necessary for a hole to be perceived as such, unless strong factors are present, a closed uniform region tends to be perceived as figure (Bozzi, 1975; Nelson \& Palmer, 2001). Factors that can specify holes unambiguously include depth cues, indicating that the enclosed region lies behind its surround and grouping factors that relate the enclosed region to a wider surround. It is also useful if figural factors like symmetry, convexity or familiarity that may lead to the perception of a figure are absent (for example, it may be difficult to see a horse-shaped region as a hole).

In practice, a few techniques have been used to study holes: e.g., random-dot stereograms (Bertamini \& Mosca, 2004; Gillam \& Cook, 2001; Qiu \& von der Heydt, 2005), kinematograms in which relative motion establishes a similar unambiguous depth order (Hulleman \& Humphreys, 2005) and real life piece of cardboard with holes cut out and freely viewed by the observers (Palmer, 1999).

For the sake of illustration, we use a landscape in Fig. 3 to discuss holes and amodal completion. We start with case A, in which the background extends not only beyond the hole region, but also beyond the object-with-hole. There is little ambiguity here because the enclosed region is related to the wider surround (Nelson \& Palmer, 2001).

Case B is more interesting because under pictorial conditions (like the printed figure), the central dotted region would not appear as a hole. Suppose, however, that binocular and motion parallax forces us to see this region as a hole. Would the dotted surface extend amodally beyond the size of the hole? This seems necessary for any compelling perception of a visual hole. The next question is the amount and direction of amodal completion. ${ }^{2}$ The reader can explore this issue by fusing the stereograms in Figs. 4 and 7. The (lack of) disparity of the dots, as well as the monocular regions of the background, makes the percep-

\footnotetext{
2 The term completion implies that the visible region is incomplete. This is obvious when T-junctions are present to signal occlusion, less obvious but still true when occlusion is specified by other factors as in the case of visual holes.
} 

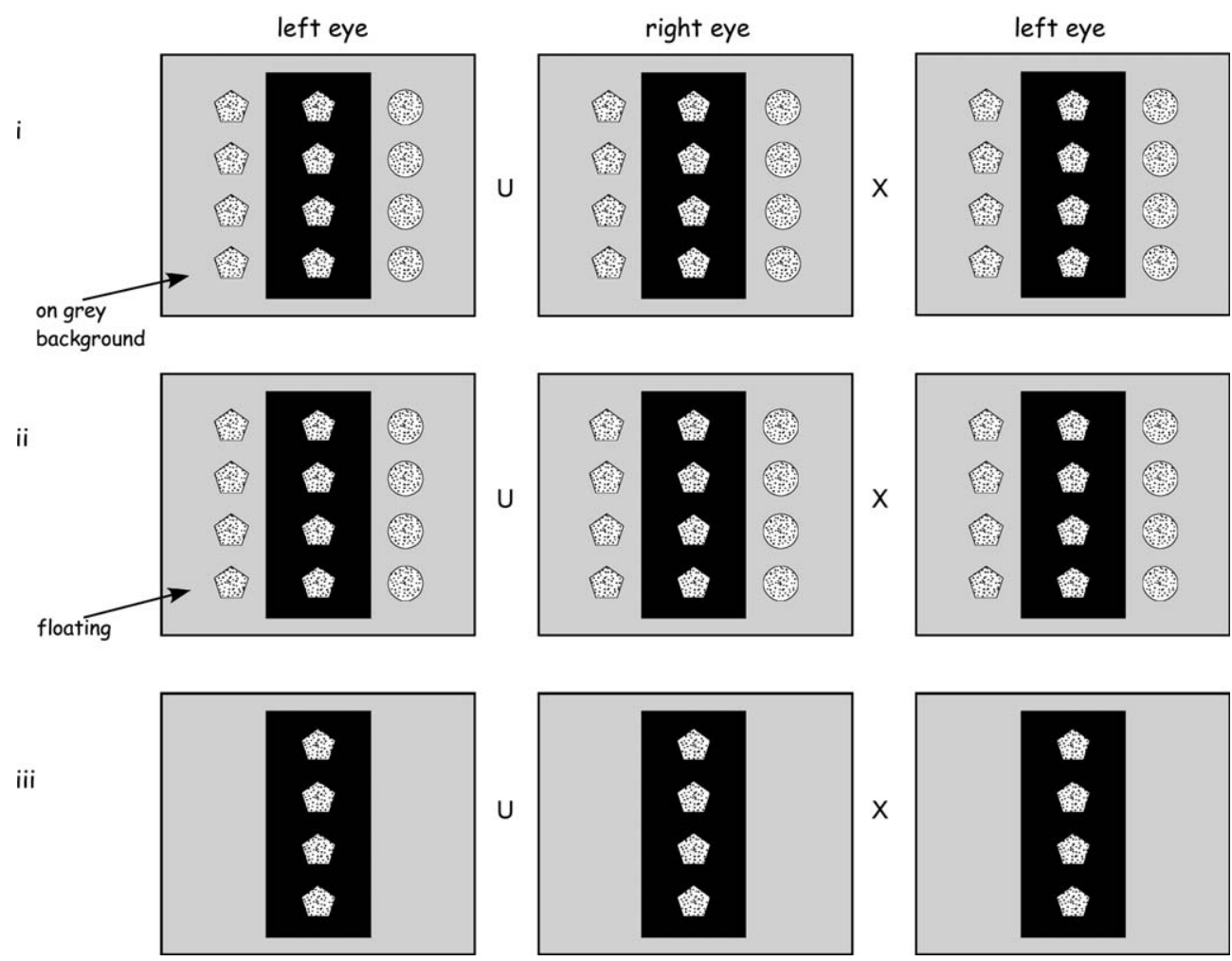

Fig. 4. In these stereograms, we specified a simple black occluder with four holes. The left and right pairs are for uncross (U) and cross (X) fusion, respectively. Nine naïve observers looked at the stereogram (i) using a stereoviewer, nine looked at (ii) and another nine looked at (iii) (total $N=27$ ). When the two images are fused, the black shapes float in front of the background and the dots seen through the holes are located on the farther surface. In (i) the pentagons and the circles are coplanar with the background, in (ii) they are coplanar with the black object, and in (iii) they are absent.

tion of hole unambiguous. ${ }^{3}$ A possibility is that the surface only extends a small distance beyond the margins of the hole. This is consistent with the idea that surfaces are minimised by the completion process (Fantoni et al., 2005) and also consistent with the idea of a partial modal completion (Brooks, Lai, \& Palmer, 2004). Extension over a small distance would be the consequence of two processes working in opposite directions: one process that extends the surface (visible through the hole) outwards, and a process that minimises this extension. If so, the amodal percept in the case of a visual hole depends on its shape,

\footnotetext{
${ }^{3}$ In the case of an object at some distance in front of a background, the monocular regions vary in size depending on the distance. It is even possible that they together include the entire region of background behind the occluder. Stereopsis based on such monocular information is also called Da Vinci stereopsis (e.g., Nakayama \& Shimojo, 1990). Similarly, the monocular regions seen through a hole can vary, and it is possible that only monocular regions are available to the observer, with no possible fusion. One can easily demonstrate this using a piece of paper with a hole and holding it in front of a far landscape (like a distant building). In such cases, the observers either see a double image or suppress one of the two views. In our stimuli, we use much smaller differences between the images of the two eyes.
} 
and examples B and $\mathrm{C}$ show two different amodal percepts (dashed lines). This is interesting, because the hole is a property of the occluder and is not connected with the background (it is on a different plane). Its effect would therefore be an effect at a distance. ${ }^{4}$

The dashed-line solution in $\mathrm{B}$ and $\mathrm{C}$ is the result of a local process but global factors can also affect the percept. In D, it is unlikely that a complex background object is perceived behind the complex hole. Instead, a globally simple region, like a blob, may be perceived. We see this issue as directly related to the distinction between local and global completion factors in the literature (Boselie, 1994; De Wit \& Van Lier, 2002; Kanizsa, 1979; Sekuler, 1994).

To explore amodal completion in relation to visual holes, we asked 27 naive observers to fuse the stereograms in Fig. 4 using a stereoviewer. In all cases, we started by asking whether the black surface was clearly perceived as in front of the grey surface. All 27 observers confirmed that that was the case and that there were four holes in it. Next, we asked them to describe what was on the grey square. We were seeking evidence about what is perceived underneath the black occluder. We hypothesised that if the shapes under the pentagonal holes had a pentagonal shape, or even just a shape more similar to a pentagon than to a circle, they would group with the pentagons on the left. If so, observers may report four circles on the right and eight angular shapes on the left. Note that Palmer, Brooks, and Nelson (2003) have already demonstrated that grouping takes place after amodal completion.

(i) Nine observers saw the first pair of stereograms. Here, the pentagons and circles are coplanar with the grey square, and so are the dots visible through the holes. All observers described the four pentagons on the left and the four circles on the right, but nobody reported a grouping of the pentagons on the left with a second set of pentagons partly hidden under the black occluder. We expected some grouping to happen if the shapes behind the occluder had any similarity with the shapes on the left. We, therefore, failed to find any support for the idea that the shape perceived under the occluder is determined by the shape of the hole. However, when we asked how many shapes were on the grey square, 5 observers reported that they saw 12 white regions with black dots ( 3 claimed that it was impossible to say and 1 reported that there was a cloud of dots under the black occluder). This is curious, as the simpler solution would be to perceive a cloud or a single white shape extending behind the four holes as, for instance, reported in Anderson, Singh, and Fleming (2002), where black regions seen through five holes merged into a single surface that extended underneath the surface with the five holes.

(ii) Nine observers saw the second pair of stereograms, in which the pentagons and circles are on the same depth plane as the black occluder. Therefore, only the dots

\footnotetext{
${ }^{4}$ The interpretation of what is present underneath a hole is a case of inference, and could be described in terms of likelihoods and prior probabilities (Kersten, Mamassian, \& Yuille, 2004). As an exercise, consider cases B and $\mathrm{C}$ (Fig. 3). Let us assume that we know that there is a rectangle underneath the occluder. Let us also assume that all rectangle orientations are equally likely, and that all rectangle sizes are equally likely. Then, underneath a vertically oriented hole the value of the posterior probability is greater for a vertically oriented rectangle. This is because for a different orientation, say a horizontal rectangle, the contour would be more likely to be visible through the hole. Every horizontal rectangle that qualifies would qualify (after rotation) as a vertical rectangle, but not vice versa. This reasoning can be extended to all polygons with a main axis of elongation.
} 
visible through the holes are located on the grey surface. This was done because if the holes have a shape similar to their complements, then it may be necessary to locate the holes as coplanar with the figures, to facilitate grouping of the eight pentagons. This condition, therefore, might support the hypothesis that observers perceive a visual hole as having a shape similar to its complement (Palmer, 1999); in our case that would be a pentagon. None of our observers reported a group of eight pentagons. However, unlike condition (i), only 2 reported four patches, whilst 6 reported the presence of a single white region behind the black occluder ( 1 could not say).

(iii) Nine observers saw the third pair of stereograms, in which only the black occluder is present. We wanted to see whether the report of four separate shapes underneath the occluder had been primed by the context in condition (i). The results were similar to those of condition (ii): only 2 observers reported four patches behind the occluder, whilst 7 reported the presence of a single white region. The difference between condition (i) and the other two conditions suggests that context plays a strong role in determining the impression of what is partly hidden. However, if context plays a role, it is striking that no observers reported pentagonal white shapes in condition (i), given that the pentagons flanking the occluder should have provided a favourable context.

To further explore the conditions under which a single object or multiple objects are reported as existing under an occluder with holes, we prepared a new set of stimuli. We used square and circular holes. In one condition there were 3 holes, in another condition there were 3 holes and 3 flanking objects next to the occluder and in the last condition there were 4 holes and 4 flanking objects. When the flanking shapes were present, there were always two sets: circles and squares (squares replacing the pentagons in Fig. 4). To maximise disparity information, we used black and white random dots as background. Moreover, we colour-coded the shapes so that the occluder was solid blue, the surface visible within the holes had red and white dots and the flanking shapes had green and white dots. This time, instead of showing only one stimulus per person, we presented a series of 48 stimuli to each naive observer ( 3 conditions, 2 shapes for the holes, 2 positions for the flanking objects with circles either to the left or the right, 2 depth planes for the flanking objects, either coplanar with the background or with the occluder, and 2 repetitions). After the 12 observers described the display and confirmed that they saw the blue occluder as floating in front of the background, they were asked what they believed would be left on the background if the blue occluder were to be removed. They were offered a forced-choice between (i) a set of round red patches; (ii) a set of square red patches; (iii) or a single large red patch.

Unlike the previous data, the depth plane of the flanking objects did not influence the responses, probably because the observers were not directed to describe such objects. A single red patch was reported $28 \%$ of the times. This response was least likely with 3 patches and 3 flanking objects $(21.9 \%)$, followed by the condition without flanking object $(27.6 \%)$, and the one with 4 patches and 4 flanking objects $(34.4 \%)$. Red square patches and round patches were reported $37.3 \%$ and $34.7 \%$ of times, respectively. Not surprisingly, square patches were rarely reported when the holes were round and vice versa. A summary of the responses is given in Fig. 5.

These observations confirm that either multiple patches, or a single patch can be reported: 8 out of 12 observers used all three categories (therefore, reporting both multiple 


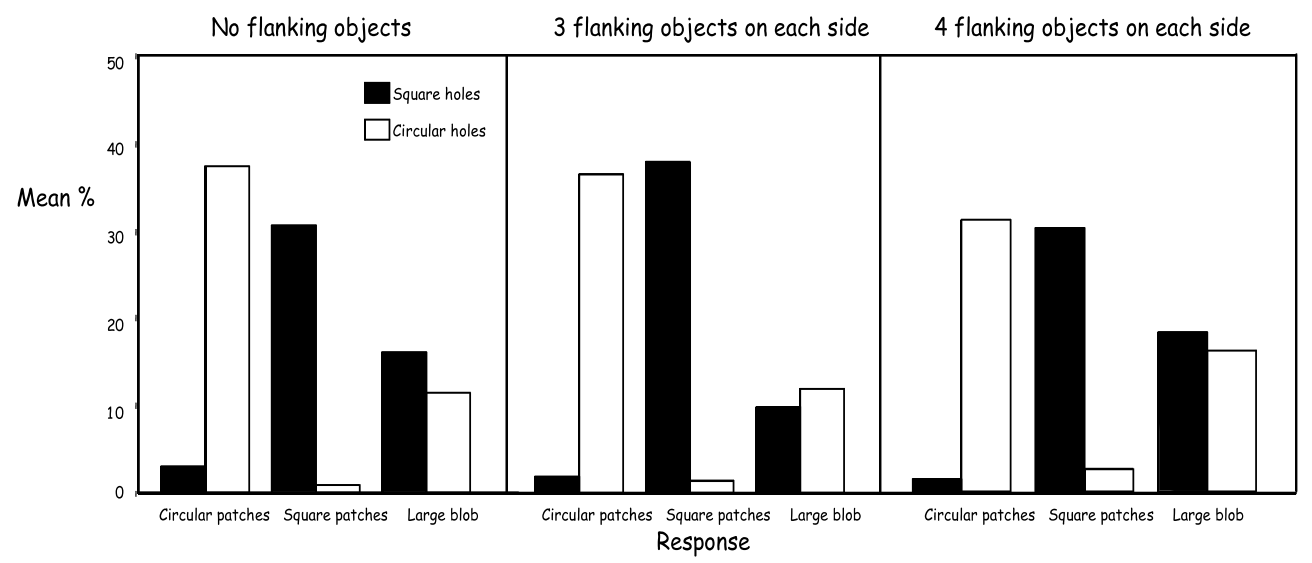

Fig. 5. The observers saw a set of 48 stimuli, and were asked for a description of what would be left if the occluder were to be removed. The graphs show the number of times (\%) in which the observers selected one of three forced-choice descriptions (Circular patches; Square patches; or Single large patch or blob) in three conditions (No flanking objects; 3 flanking objects on each side; 4 flanking objects on each side), separately for stimuli in which the holes were square (black bars) or round (white bars).

patches and a single patch). Several factors may be important. Among them are the proximity of the holes (nearer holes require less amodal completion for the patches to join), the shape of the holes (the vertical sides of square holes may have contributed to the report of a single region) and the context (presence of multiple flanking objects).

\section{Moving holes}

Binocular parallax and motion parallax are both factors that may define visual holes. They both require some kind of texture on the surface and random-dot stimuli are useful to this end. To be more precise, in the case of random-dot stereograms both binocular disparity and unpaired background features (Gillam \& Cook, 2001; Gillam \& Nakayama, 1999) contribute to the unambiguous perception of holes. In the case of random-dot kinematograms, probably the most powerful factor is the accretion and deletion of the texture caused by the displacement (Gibson et al., 1969). Gibson and collaborators studied the perception of occlusion and disocclusion and concluded that the information about texture and shape present in the stimuli was so powerful that there was no need to rely on memory or inference (Gibson et al., 1969; Kaplan, 1969; but see Wagemans \& d'Ydewalle, 1989).

We created conditions that allowed us to test what happens when a hole moves independently from the foreground texture. An explanation of various possible scenarios is given in Fig. 6. In all these examples, the green surface is specified as in front by binocular information and the palm beach scene is specified as farther back by binocular information. The actual stimuli were random-dot surfaces as shown in Fig. 7, but we use different images in Fig. 6 for clarity. In the case of random-dot stereograms, no region exists until after the left and the right images are fused or a region starts moving. We use $\mathrm{O}$ and $\mathrm{H}$ to refer to the object and the hole and we use $\mathrm{F}$ and $\mathrm{B}$ to refer to the foreground and the background as specified by binocular disparity. Finally, we use a + sign to signify displacement of the texture. Because the displays always contained a moving item (i.e., hole or object), displace- 

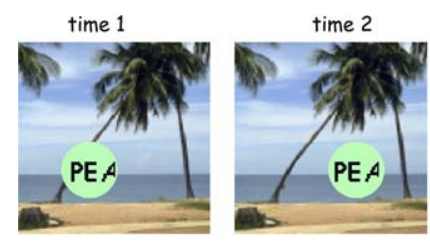

$\mathrm{OBF}+$

Background texture stationary. Foreground texture moving. Natural event where texture] moves in front of stationary background
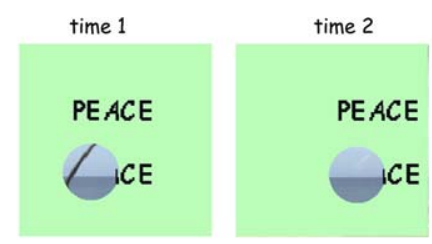

$\mathrm{HBF}$ -

Background texture stationary Foreground texture moving. Natural event where the surface-with-hole moves in front of stationary background. Hole does not change position on foreground suface. Texture foreground surface. Texture
visible through hole changes
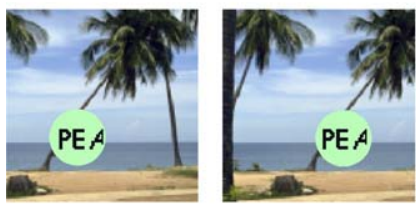

$\mathrm{OB}+\mathrm{F}+$

Background texture moving. Foreground texture moving. Natural event where the object [with unchanging texture] moves in front of a moving background (together)
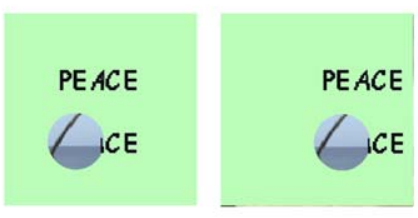

$\mathrm{HB}+\mathrm{F}+$

Background texture moving. Foreground texture moving. Natural event where the surface-with-hole moves in front of a moving background (together) Hole does not change position on foreground surface. Texture visible through hole does not change
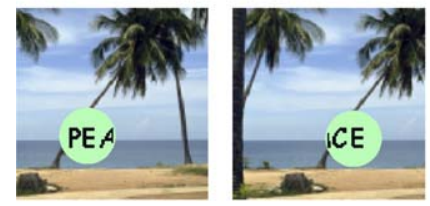

$O B+F$

Background texture moving. Foreground texture stationary. Strange event where the object changes texture while moving, (cue conflict - dispar ity versus accretion/deletion)

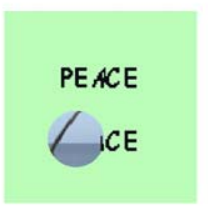

$\mathrm{HB}+\mathrm{F}$
Background texture moving. Foreground texture stationary Strange event where the hole changes position on the surface-with-hole in front of moving background. Foregroun changes shape (cue conflict disparity vs accretion/ deletion). Texture visible through hole does not change
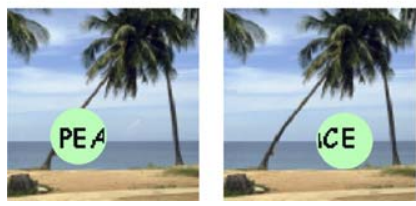

$\mathrm{OBF}$
Background texture stationary. Foreground texture sta tionary. Strange event where the object changes texture while moving.

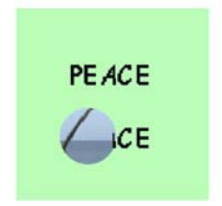
HBF

Background texture stationary Foreground texture stationary Strange event where the hole changes position on the surface-with-hole in front of a stationary background Forestationary background Foreground changes shape. Texture
visible through hole changes.

Fig. 6. A classification of events in which binocular information and texture accretion/deletion specify the presence of an item perceived either as an object or as a hole. Here we use two images (a beach scene and a solid colour surface) to explain the stimuli, but the observers saw random-dot stereograms. The solid colour surface with words (foreground) is always specified as in front of the beach scene by binocular information, independently from texture accretion/deletion. In our stimuli, the foreground was always the zero disparity plane. We use code names in which $\mathrm{F}$ and $\mathrm{B}$ refer to foreground and background, $\mathrm{O}$ and $\mathrm{H}$ refer to object and hole, and $\mathrm{a}+$ sign refers to displacement of texture. The legend in the figure explains that in cases $\mathrm{OB}+\mathrm{F}, \mathrm{OBF}, \mathrm{HBF}+\mathrm{and} \mathrm{HBF}$ there is a cue conflict. (For interpretation of the references to colour in this figure legend, the reader is referred to the web version of this article.)

ment of the texture in the disparity plane of the moving item (foreground for an object and background for a hole) results in the texture travelling with the item, so the texture will not move relative to the item (like the stripes of a zebra when the zebra moves). Similarly, nondisplacement of the texture in the disparity plane of the moving item results in the texture moving relative to the item. So, a + sign in the item plane indicates that the texture is attached to the item. Absence of a + sign in the item plane indicates that the texture of the item changes from frame to frame.

For four of the conditions in Fig. $6(\mathrm{OBF}+\mathrm{OB}+\mathrm{F}+, \mathrm{HB}+\mathrm{F}+$, and $\mathrm{HBF}+)$, the situation should be clear: in $\mathrm{OBF}+$ and $\mathrm{OB}+\mathrm{F}+$ a textured object moves in front of a background with stationary $(\mathrm{B})$, or moving texture $(\mathrm{B}+)$, respectively. Only in case $\mathrm{OBF}+$ there is accretion and deletion, because the texture on the background is not moving, hence, is covered and uncovered by the moving object. In case $\mathrm{OB}+\mathrm{F}+$ there is no accretion or deletion, because the texture on the background moves with the same velocity as the moving object, so the part of the background that is covered by the object stays the 

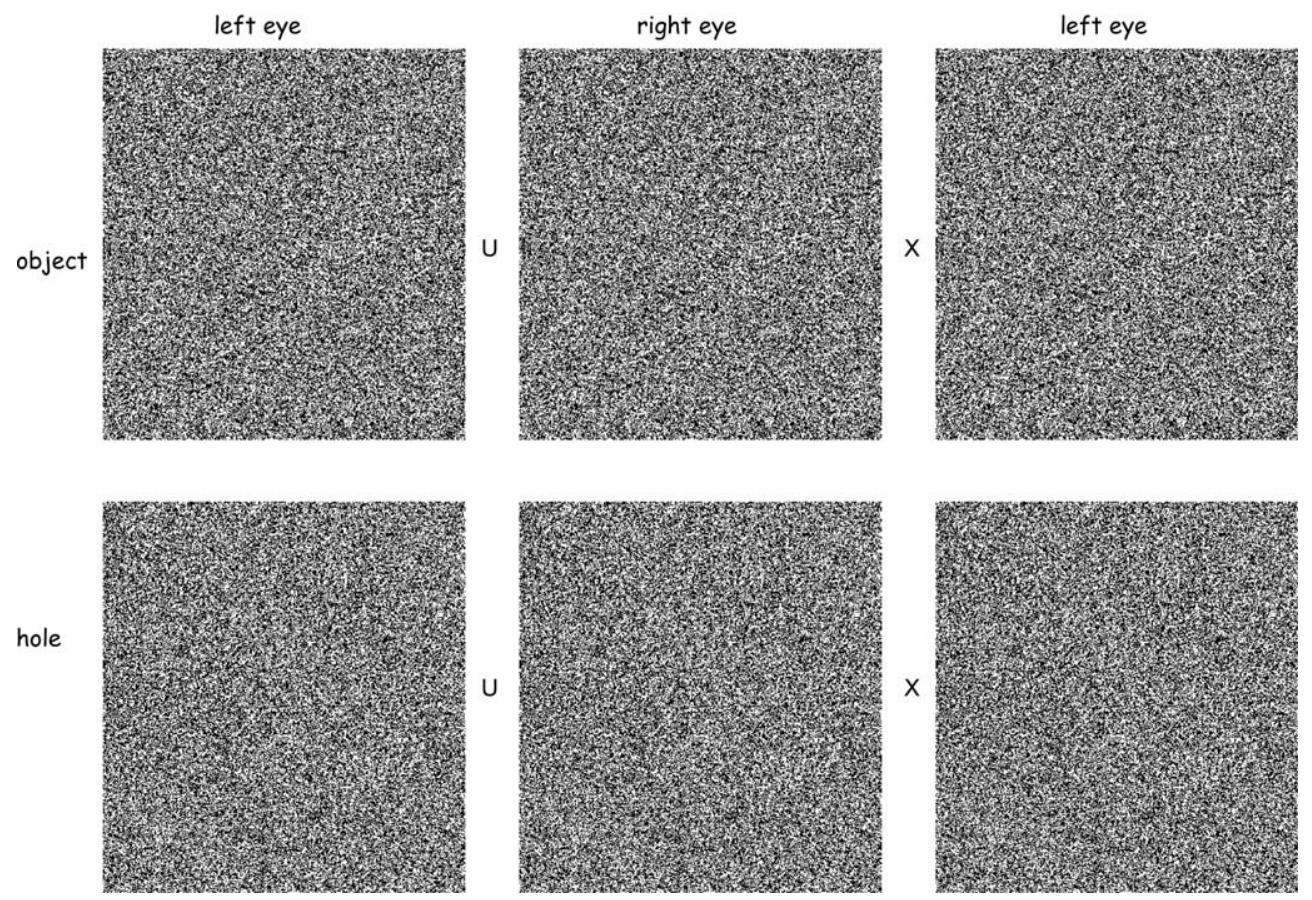

Fig. 7. Random-dot stereograms of figures and holes shown to the observers. The left and right pairs are for uncross (U) and cross (X) fusion, respectively. These stimuli were used to create the conflict situations described in Fig. 3 but it is not possible to show both binocular information and motion in a static picture. To help the readers to fuse these images, we have cropped a large portion of the surround (from $800 \times 600$ to $370 \times 400$ ). Animations in which we replaced black and white dots in one depth plane with red and white dots are also available from: http://www.liv.ac.uk/vp/michotte.html.

same from frame to frame. In $\mathrm{HB}+\mathrm{F}+$ and $\mathrm{HBF}+$, an object-with-hole is moving in front of the background with moving $(\mathrm{B}+)$, or stationary texture $(\mathrm{B})$, respectively. In case $\mathrm{HBF}+$, there is accretion and deletion inside the hole, because the texture on the background is not moving. So, as the object-with-hole moves, different parts of the background texture become visible through the hole. In case $\mathrm{HB}+\mathrm{F}+$, the background texture that is visible through the hole stays the same for all the frames, because the background texture is moving with the same velocity as the object with hole. In all these four cases, there is no accretion/deletion in the foreground (as defined by disparity). We expect this to be a critical factor in determining the percept. Burge, Peterson, and Palmer (2005) have recently reported that ordinal figure/ground cues (accretion/deletion is information of this type) interact with metric depth cues. They reported that familiarity influenced the perceived depth as defined by the disparity of random dots. When both disparity and familiarity specified that a surface was closer, the perceived depth was greater than when a conflict was present.

The other four conditions are therefore more interesting. In cases $\mathrm{OB}+\mathrm{F}$ and $\mathrm{OBF}$, there is accretion at the leading edge and deletion at the trailing edge on the figural side of the contour. This corresponds to a moving object that changes its texture. From frame to frame, a texture element on the object will move in the direction opposite to the direction 
of motion of object. Cases $\mathrm{HB}+\mathrm{F}$ and $\mathrm{HBF}$ correspond to a hole changing its location on the object-with-hole. Here, there now is deletion at the leading edge and accretion at the trailing edge, because as the hole moves, it covers and uncovers texture of the object-withhole. In case $\mathrm{HBF}$, there are accretion and deletion cues inside the hole as well: as the hole is moving, it is uncovering new parts of the background. In case $\mathrm{HB}+\mathrm{F}$, the information visible through the hole stays identical over the period in which the hole is moving. Here, there are no accretion and deletion cues inside the hole. If the contour belongs to the figural side (as defined by disparity), we would expect ambiguities in the perception of the moving item for all four cases because accretion and deletion have been introduced at the figural side of the contour. If holes do own the contour as well, then HBF might prove to be an exception: here, the owner of the contour moves with the contour. Similarly, case $\mathrm{HB}+\mathrm{F}$ might still be perceived as a hole. However, because there are no accretions and deletions inside the hole anymore, it might be less obvious that case $\mathrm{HB}+\mathrm{F}$ is a moving hole.

\subsection{Method}

\subsubsection{Participants}

The 16 participants were (postgraduate) members of staff in the Department of Psychology at the University of Hull. Nine were female, seven were male. All of them had normal or corrected to normal vision and none of them were aware of the purpose of the study. The participants all passed a test of stereovision.

\subsubsection{Stimuli and design}

The experiment was controlled by a $3 \mathrm{GHz}$ Pentium IV PC with an NVIDIA Geforce 6800 graphics adapter. The stimuli were presented on a 19 in. Iiyama HM903DTB monitor in $800 \times 600(120 \mathrm{~Hz})$ graphics mode. NuVision $60 \mathrm{GX}-\mathrm{NSR}$ shutter glasses were used to present stereo images with effective refresh rate of $60 \mathrm{~Hz}$ for each eye.

Stimuli for the left and right eyes consisted of a combination of two independently generated random pixel arrays. The arrays were presented at $0^{\circ}$ and $0.2^{\circ}$ disparity, creating a foreground and a background plane. For the object displays, a $4.5^{\circ}$ circular area containing pixels from the foreground plane was combined with a surround containing pixels from the background plane. For the hole displays, the same $4.5^{\circ}$ circular area contained pixels from the background plane and was combined with a surround drawn from the foreground plane.

The item (i.e., object or hole) was always moving from left to right with a speed of $6 \%$ s. The motion of the pixels was manipulated independently from the motion of the item. The pixels in the plane in which the item (object/hole) was drawn were either moving to the right with $6 \%$, resulting in a display where the texture on the item was identical for all the frames, or the pixels would be stationary. This would result in the texture of the item changing from one frame to the next, because the texture on the item would be acquired from different parts of the random pixel array. The motion of the pixels in the surrounding plane was manipulated as well. There were 120 frames per stimulus sequence. Consequently, each stimulus sequence lasted for $1 \mathrm{~s}$.

There were three factors: item (either an object or a hole); motion of foreground plane $(0 \% \mathrm{~s}$ or $6 \% \mathrm{~s})$; and motion of the background plane $(0 \% \mathrm{~s}$ or $6 \% \mathrm{~s})$. These three factors were fully crossed, resulting in a total of 8 conditions as described in Fig. 6. 


\subsubsection{Procedure}

In every trial, the stimulus sequence started with the item (object/hole) stationary for $2 \mathrm{~s}$. After this period, the items started to move to the right with a speed of $6 \%$. After the motion ended, the item remained on the screen in its final position. The item started with the $x$-position of its centre $5.4^{\circ}$ to the left of the vertical midline of the display. The $y$-position of the centre was varied randomly in a zone of $9^{\circ}$, centred on the horizontal midline of the display. The task of the participants was to describe what they saw and the experimenter recorded their description. The participants were able to repeat the sequence as often as they liked and a new trial was only started when the participants indicated that they were satisfied with the description that they had provided.

The four trials with consistent disparity and accretion/deletion cues were presented first. The four trials with inconsistent cues were presented last. Each participant received a different trial sequence.

\subsection{Results}

As expected, all of the participants agreed on their interpretations of the four displays without accretion/deletion in the foreground. They reported a disc in front of a background for displays $\mathrm{OBF}+$ and $\mathrm{OB}+\mathrm{F}+$, and a circular hole in the foreground through which a background was visible for displays $\mathrm{HB}+\mathrm{F}+$ and $\mathrm{HBF}+$. They also agreed in their descriptions of displays $\mathrm{OB}+\mathrm{F}, \mathrm{OBF}, \mathrm{HB}+\mathrm{F}$, and $\mathrm{HBF}$ as long as the item was not moving. Furthermore, all of them noticed that displays with accretion/deletion in the foreground (the second set of four trials) were qualitatively different from the displays they had seen up till then. However, the descriptions of their percept as soon as the item had started to move in displays $\mathrm{OB}+\mathrm{F}, \mathrm{OBF}, \mathrm{HB}+\mathrm{F}$ and $\mathrm{HBF}$ diverged considerably from the descriptions of the other four displays (see Fig. 8).

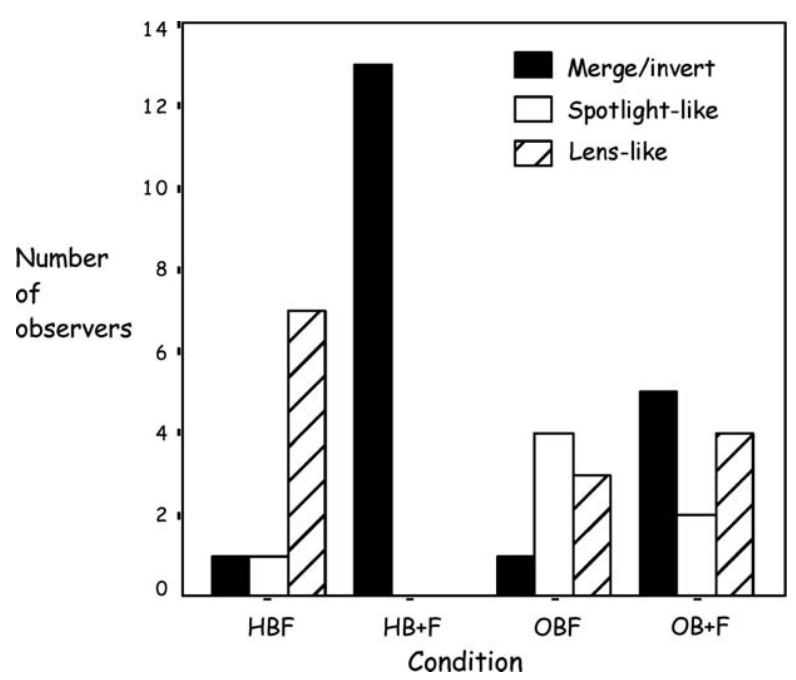

Fig. 8. The number of observers reporting merging, and/or inversion of the depth planes (black); a spotlight-like appearance of the moving item (white) and a lens-like appearance of the moving item (hatched), as a function of stimulus condition. 
A large proportion of participants reported a change in the depth ordering of the planes (either the two depth planes merging into a single plane, or even an inversion of the depth ordering) for displays $\mathrm{HB}+\mathrm{F}$ (13 out of 16 participants) and $\mathrm{OB}+\mathrm{F}$ (5 out of 16 participants). However, this was not the only change due to the introduction of accretion and deletion cues in the foreground: observers reported a lens-like appearance, where it seemed that they were seeing the texture on the moving item through a (magnifying) glass $(4 / 16,3 /$ 16 and 7/16, for displays $\mathrm{OB}+\mathrm{F}, \mathrm{OBF}$ and $\mathrm{HBF}$, respectively), or a spotlight-like appearance $(2 / 16,4 / 16$ and $1 / 16$, for displays $\mathrm{OB}+\mathrm{F}, \mathrm{OBF}$, and $\mathrm{HBF}$, respectively). So, for at least half of the observers (one subject reported depth inversion for displays OBF and HBF), there is a large perceptual change when accretion/deletion is introduced on the figural side of a moving contour. Importantly, observers found it difficult to perceive a moving hole in display HBF, even though the hole contained accretion and deletion cues on the inside.

It seems that the owner of the contour has to move with the contour and that a hole does not qualify as owner (at least not under the conditions that we have used in this experiment. Parametric manipulation would be necessary to establish the generality of this observation). This would explain why almost half of the observers reported a lens-like structure during the motion of HBF: they introduced an owner for the contour that could move with the contour. An important property of a lens is that the texture does not belong to the visible contour in which it finds itself. In this way, there can be accretion and deletion on the figural side of a contour, because the accretion and deletion of texture visible through a lens are not occurring at the figural depth. A similar argument would hold for a spotlight. Note that the introduction of accretion and deletion on the figural side affects both objects and holes. There is no principled difference between the introduction of a transparent object or making an object go transparent. For the hole, the transparency was already in place and only needed to be assigned to an object, whereas for the object, the object was already in place and only needed to become transparent.

What we may be observing in $\mathrm{OB}+\mathrm{F}, \mathrm{OBF}, \mathrm{HB}+\mathrm{F}$, and $\mathrm{HBF}$ is a confusing type of percept that is the result of an attempt by the system to create an appropriate stratification of the stimulus. We believe there are static parallels to this situation. van Lier, De Wit, and Koning (2006) have studied a display in which illusory Kanizsa squares are positioned on top of a background grid. When the illusory contours and physical contours are misaligned, the resulting percept appears to be rather remarkably unstable. It is as if the illusory square appears to deform the bars seen underneath it. Other examples that are related to this phenomenon can be found in Bozzi (1975). Bozzi observed how the misalignment of contours can generate a sense of transparency in the absence of any of the conditions usually associated with transparency. What is shared by these stimuli and our stimuli is not just a "restless appearance" (van Lier et al., 2006), there is also a paradoxical stratification: if the illusory square in their displays is transparent, we should be able to see the bars as well as the complete circles underneath the square (but we only see the bars). Similarly, in HBF the lens percept solves the problem of independent motion of the item because a lens, unlike a hole, is not a feature of the object, yet if the lens is in front, we should see the foreground behind it.

\section{General discussion}

In this paper, we have explored the appearance of surfaces seen through visual holes. This is a special case of amodal completion in which contour information is absent. We 
found no support for the idea that the surface behind a hole takes on the shape of the complement of the hole (e.g., the pentagon in the examples in Fig. 4). Observers found it difficult to judge the extension of the surface for which there was no contour information. Perhaps not surprisingly, given this fundamental uncertainty, the context played a role. When 4 patches were present on each side of the occluder the observers reported that there were 4 patches behind the holes (one for each hole), but when the context was absent (or located on a different depth plane), the observers tended to report a single surface extending behind the holes. A similar effect of context can be seen when comparing the first two conditions in Fig. 5, which differ only on the presence of flanking objects. The role of context in the literature has been debated in relation to where amodal completion finds itself on the perception/cognition continuum (Gerbino \& Salmaso, 1987; Kanizsa \& Gerbino, 1982). Recently, the role of context has been re-evaluated in relation to its importance in a visual search task (Rauschenberger, Mosca, Peterson, \& Bruno, 2004).

In another set of observations, we created holes that were moving independently of the object-with-hole. If the hole owns the contour it shares with the object-with-hole, a hole moving independently should be acceptable, because the accretion/deletion is added to the non-owned side of the contour. However, a substantial proportion of participants perceived a lens in the aperture of the hole. When accretion and deletion were introduced at the figural side of objects, the response was similar: detachment of the contour from the texture inside the contour. This resulted in a spotlight or lens-like appearance for the object.

There seems to be a clear resistance to perceive holes moving independently from the object-with-hole. The results in Section 3 support the view that the contour of a hole belongs to the object-with-hole. They suggest that contour ownership is incompatible with accretion/deletion on the figural side of the contour. All four cases where there was accretion/deletion on the figural side resulted in detachment of texture, and introduction of a lens-like/spotlight-like appearance. If contour ownership is incompatible with accretion/ deletion, it means that the contour is owned by the object-with-hole in case HBF+ as well. Here, all observers clearly saw a moving hole, even though there were accretion and deletion inside the hole. But because there is no accretion/deletion on the side of the owner of the contour (the object-with-hole), there is no need to detach the texture. We conclude that even in static situations, the contour of the hole is not owned by the hole region. This goes against the suggestion made by Palmer (1999) and supports the principle that the hole is a shape property of the object-with-hole (Bertamini, in press).

Our results have implications for two phenomena that were studied by Michotte: the tunnel effect (Michotte et al., 1967), and the perception of causality (Michotte, 1946). The tunnel effect is the continuous experience of an object's movement, even though its middle phase takes place behind an occluder (Burke, 1952; Michotte et al., 1967). The literature has focused on the fact that the same object is perceived as emerging from behind the occluder, even when the object changes appearance, for instance in colour or shape (Carey $\& \mathrm{Xu}, 2001$ ). Kawachi and Gyoba (2006) found indirect evidence of object permanence using a speeded response task.

We believe that a hole is a property of the object-with-hole. Because the object-withhole will not go out of existence, we expect a more compelling tunnel-effect. Rather than the owner of the contour going out of existence, there is merely a part of the object-withhole that becomes occluded. In this sense, an occluded hole is conceptually less related to the tunnel effect and more to the dynamic occlusion studied, for instance, by Wagemans and d'Ydewalle (1989). For the special case of a hole moving independently from its object, 
we expect a tunnel effect. However, this is because a transparent object has been introduced and it is this object that goes out of existence, rather than the hole.

A difference between objects and holes should occur in the case of perception of causality. As is well known from Michotte's classic work (Michotte, 1946), the percept of causality emerges directly from a visual event given the appropriate spatio-temporal parameters (see also the discussion of causality by White, 2006). However, consider the case of a hole moving and when it stops a second hole starts moving. When the holes move, they move with the object-with-hole and they are just a feature of that object; therefore, the fact that the object-with-hole translates is sufficient to explain the motion of the holes. No causal relation between the two holes is necessary. Clearly the necessary comparison would be a case in which objects move and the background moves as well. This background could explain the motion of the objects if one imagines that the objects are attached to it but background and objects are separate entities. By contrast the object-with-hole and the holes cannot be separate entities. In sum, holes cannot play the independent role that is played by objects and this has clear perceptual consequences.

The phenomenological method allows exploration and insight into amodal completion, but it should be complemented by other methods (Pomerantz \& Kubovy, 1981), and some nice examples are included in this special issue (Choi \& Scholl, 2006; Kawachi \& Gyoba, 2006). We are currently using holes to study priming across figure-ground reversals, the (absence of) underlying medial axis representation of ground regions, and the role of concavities to guide attention. We expect holes to play a central role in helping to answer important questions related to figure-ground organisation and shape representation.

\section{References}

Anderson, B. L., Singh, M., \& Fleming, R. W. (2002). The interpolation of object and surface structure. Cognitive Psychology, 44, 148-190.

Bertamini, M. (in press). Who owns the contour of a visual hole? Perception.

Bertamini, M., \& Croucher, C. J. (2003). The shape of holes. Cognition, 87(1), 33-54.

Bertamini, M., \& Lawson, R. (2006). Visual search for a circular region perceived as a figure versus as a hole: evidence for the importance of part structure. Perception \& Psychophysics.

Bertamini, M., \& Mosca, F. (2004). Early computation of contour curvature and part structure: Evidence from holes. Perception, 33, 35-48.

Boselie, F. (1994). Local and global factors in visual occlusion. Perception, 23, 517-528.

Bozzi, P. (1975). Osservazioni su alcuni casi di trasparenza fenomenica realizzabili con figure a tratto [Observations on some cases of phenomenal transparency using line drawings]. In G. B. Flores D'Arcais (Ed.), Studies in perception: Festschrift for Fabio Metelli (pp. 88-110). Aldo Martello: Milan.

Brooks, J. L., Lai, K., \& Palmer, S. E. (2004). The occlusion illusion: modal completion or apparent distance? Journal of Vision, 4, 722a [Abstract].

Burge, J., Peterson, M. A., \& Palmer, S. E. (2005). Ordinal configural cues combine with metric disparity in depth perception. Journal of Vision, 4, 534-542.

Burke, L. (1952). On the tunnel effect. Quarterly Journal of Experimental Psychology, 4, 121-138.

Carey, S., \& Xu, F. (2001). Infants' knowledge of objects: beyond object files and object tracking. Cognition, 80, $179-213$.

Casati, R., \& Varzi, A. C. (1994). Holes and other superficialities. Cambridge, MA: MIT Press.

Choi, H., \& Scholl, B. J. (2006). Measuring causal perception: Connections to representational momentum? Acta Psychologica, 123, 91-111.

De Wit, T. C. J., \& Van Lier, R. J. (2002). Global visual completion of quasi-regular shapes. Perception, 31, 969984.

Fantoni, C., Bertamini, M., \& Gerbino, W. (2005). Contour curvature polarity and surface interpolation. Vision Research, 45, 1047-1062. 
Fantoni, C., \& Gerbino, W. (2003). Contour interpolation by vector-field combination. Journal of Vision, 3, 281303.

Fulvio, J. M., \& Singh, M. (2006). Surface geometry influences the shape of illusory contours. Acta Psychologica, $123,20-40$.

Gerbino, W., \& Salmaso, D. (1987). The effect of amodal completion on visual matching. Acta Psychologica, 65, 25-46.

Gibson, J. J., Kaplan, G. A., Reynolds, H. E. N., \& Wheeler, K. (1969). The change from visible to invisible: a study of optical transitions. Perception \& Psychophysics, 5, 113-116.

Gillam, B., \& Cook, M. L. (2001). Perspective based on stereopsis and occlusion. Psychological Science, 12, 424 429.

Gillam, B., \& Nakayama, K. (1999). Quantitative depth for a phantom surface can be based on cyclopean occlusion cues alone. Vision Research, 39, 109-112.

Hulleman, J., \& Humphreys, G. W. (2005). The difference between searching amongst objects and searching amongst holes. Perception \& Psychophysics, 67, 469-482.

Kanizsa, G. (1979). Organization of vision. New York: Praeger.

Kanizsa, G., \& Gerbino, W. (1982). Amodal completion: seeing or thinking? In J. Beck (Ed.), Organisation and representation in perception (pp. 167-190). Hillsdale, NJ: Erlbaum.

Kaplan, G. (1969). Kinetic disrupture of optical texture: the perception of depth at an edge. Perception \& Psychophysics, 6, 193-198.

Kawachi, Y., \& Gyoba, J. (2006). A new response-time measure of object persistence in the tunnel effect. Acta Psychologica, 123, 73-90.

Kellman, P. J., Guttman, S. E., \& Wickens, T. D. (2001). Geometric and neural models of object perception. In T. Shipley \& P. J. Kellman (Eds.), From fragments to objects: Segmentation and grouping in vision (pp. 183-245). Oxford: Elsevier.

Kersten, D., Mamassian, P., \& Yuille, A. (2004). Object perception as Bayesian inference. Annual Review of Psychology, 55, 271-304.

Kubovy, M., \& Gepshtein, S. (2003). Grouping in space and in space-time: an exercise in phenomenological psychophysics. In M. Behrmann \& R. Kimchi (Eds.), Perceptual organization in vision: Behavioral and neural perspectives (pp. 45-85). Mahwah, NJ: Erlbaum.

Michotte, A. (1946). La perception de la causalité. Louvain: Publications Universitaires.

Michotte, A. (1950). A propos de la permanence phénoménale: Faits et théories. Acta Psychologica, 7, 298-322.

Michotte, A., Thinès, G., \& Crabbé, G. (1967). Les compléments amodaux des structures perceptives. Louvain: Publications Universitaires.

Nakayama, K., \& Shimojo, S. (1990). Da Vinci stereopsis: depth and subjective occluding contours from unpaired image points. Vision Research, 30,1811-1825.

Nakayama, K., Shimojo, S., \& Silverman, G. H. (1989). Stereoscopic depth: its relation to image segmentation, grouping, and the recognition of occluded objects. Perception, 18, 55-68.

Nelson, R., \& Palmer, S. E. (2001). Of holes and wholes: the perception of surrounded regions. Perception, 30 , 1213-1226.

Palmer, S. E. (1999). Vision science: Photons to phenomenology. Cambridge, MA: MIT Press.

Palmer, S. E., Brooks, J. L., \& Nelson, R. (2003). When does grouping happen? Acta Psychologica, 114, 311-330.

Peterson, M. A. (2003). On figures, grounds, and varieties of surface completion. In R. Kimchi, M. Behrmann, \& C. Holson (Eds.), Perceptual organization in vision: Behavioral and neural perspectives (pp. 87-116). Mahwah, NJ: Erlbaum.

Pomerantz, J. R., \& Kubovy, M. (1981). Perceptual organization: an overview. In M. Kubovy \& J. R. Pomerantz (Eds.), Perceptual organization (pp. 423-456). Hillsdale, NJ: Erlbaum.

Qiu, F. T., \& von der Heydt, R. (2005). Figure and ground in the visual cortex: V2 combines stereoscopic cues with Gestalt rules. Neuron, 47, 155-166.

Rauschenberger, R., Mosca, F., Peterson, M. A., \& Bruno, N. (2004). Amodal completion in visual search. Preemption or context effects? Psychological Science, 15, 351-355.

Sekuler, A. B. (1994). Local and global minima in visual completion: effects of symmetry and orientation. Perception, 23, 529-545.

Shimojo, S., \& Nakayama, K. (1990). Amodal presence of partially occluded surfaces: role of invisible stimuli in apparent motion correspondence. Perception, 19, 285-299.

Shipley, T., \& Kellman, P. (1994). Spatiotemporal boundary formation: boundary, form and motion perception from transformations of surface elements. Journal of Experimental Psychology: General, 123, 3-20. 
van Lier, R. J., De Wit, T. C. J., \& Koning, A. R. (2006). Con-fusing contours \& pieces of glass. Acta Psychologica, $123,41-54$.

van Lier, R. J., \& Wagemans, J. (1999). From images to objects: global and local completions of self-occluded parts. Journal of Experimental Psychology: Human Perception and Performance, 25, 1721-1741.

Wagemans, J., \& d'Y dewalle, G. (1989). The effects of kinetic occlusion and categorization on amodal completion. Acta Psychologica, 72, 281-293.

White, P. A. (2006). The role of activity in visual impressions of causality. Acta Psychologica, 123, $166-185$.

Wuerger, S., Shapley, R., \& Rubin, N. (1996). "On the visually perceived direction of motion" by Hans Wallach: 60 years later. Perception, 25, 1317-1367.

Yin, C., Kellman, P. J., \& Shipley, T. F. (2000). Surface integration influences depth discrimination. Vision Research, 40, 1969-1978. 